\title{
Absolute Quantification for Small-Animal PET
}

\author{
Vincent Keereman, Roel Van Holen, Christian Vanhove, Pieter Mollet, Stefaan Vandenberghe
}

\begin{abstract}
Quantification is important in preclinical PET studies. To achieve absolute quantification, an accurate reconstruction algorithm is necessary. Such an algorithm includes corrections for different effects such as geometric sensitivity of the scanner, detection efficiency, attenuation, scatter and random coincidences. In this work we present a method for performing absolute quantification on the LabPET system.

All acquisitions were done on a GE Triumph system. This tri-modality system consists of a micro-PET (LabPET), microCT (X-O) and micro-SPECT (X-SPECT) scanner. Three PET scans were done. In the first scan 5 vials with different activity concentrations of ${ }^{18}$ F-FDG were scanned. The total activity inside the scanner was $80 \mathrm{MBq}$. The second scan was performed after 4 hours when the total activity in the scanner had decayed to 20 MBq. In the third scan 3 vials and 1 sphere were scanned with a total activity of $20 \mathrm{MBq}$. Before each PET scan a micro-CT scan was acquired.

Point sources with a known activity were placed inside the field of view. The counts obtained in these point sources are used to obtain a correction factor for absolute sensitivity. Reconstruction was done using a 3D ML-EM reconstruction with micro-CT based attenuation correction. VOIs were drawn over the vials and the sphere in the reconstructed images. The total activity in the VOIs was calculated using the correction factor for absolute sensitivity. It was compared to the activity measured in a dose calibrator.

The average quantification error was $56 \%, 6.4 \%$ and 0.6 $\%$ for the first, second and third scan. The high error in the first scan is explained by count rate effects, as $80 \mathrm{MBq}$ can be considered a high activity level for this system.

The feasibility of absolute quantification on the LabPET system was demonstrated. When the count rate is below $20 \mathrm{MBq}$ absolute quantification is possible with an average quantification error smaller than $6.4 \%$.
\end{abstract}

Index Terms-micro-PET, micro-CT, quantification, attenuation correction.

\section{INTRODUCTION}

$\mathbf{I}$ $\mathrm{N}$ clinical imaging, most PET studies are used for lesion detection. A qualitative interpretation of PET images is sufficient for this task. However, quantitative imaging is also possible with PET. The advantage of quantitative PET has been demonstrated in clinical studies, especially for evaluation of the response to chemotherapy or radiotherapy[1], [2]. Quantitative PET images are also needed for kinetic modeling of tracer uptake[3].

Quantification is also important in small-animal PET studies. As mentioned above, lesion detection is still the most important application of clinical PET. However, the lesion location is often well known in preclinical PET studies. This is, for example, the case in growth studies of inoculated

V. Keereman, R. Van Holen, C. Vanhove, P. Mollet and S. Vandenberghe are with Department of Electronics and Information Systems, MEDISIP, Ghent University-IBBT-IBiTech, De Pintelaan 185 Block B, 9000 Gent, Belgium (email: Vincent.Keereman@ugent.be)
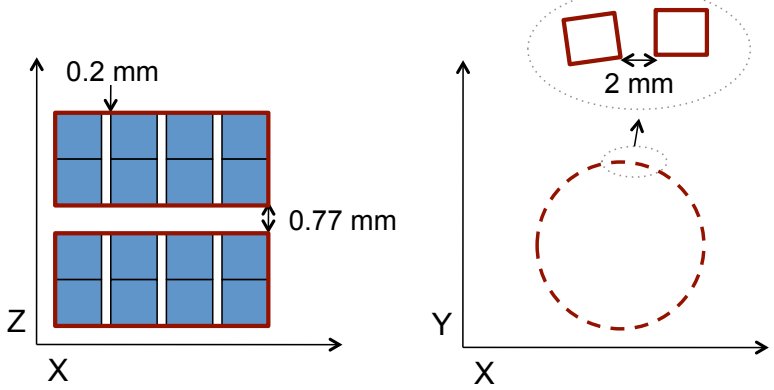

Figure 1. Illustration of the gaps in the LabPET ${ }^{\mathrm{TM}}$ scanner. On the left two modules are shown (red line), each containing 4 × 2 crystals (blue). The crystals are $2 \times 2 \mathrm{~mm}$. There are $0.77 \mathrm{~mm}$ gaps between all modules in the axial direction. Within each module, there are $0.2 \mathrm{~mm}$ gaps between all crystals in the tangential direction. On the right, the full ring is illustrated with an inset illustrating the gap between two modules in the tangential direction, which is approximately $2 \mathrm{~mm}$.

tumors[4]. The researcher is then only interested in quantifying the uptake of the lesion as accurately as possible. Absolute quantification is also of interest to researchers performing longitudinal preclinical studies, in which the activity uptake in different regions needs to be compared between different time points[5].

To achieve absolute quantification, an accurate reconstruction algorithm is needed. Such an algorithm includes corrections for different effects such as geometric sensitivity of the scanner, detection efficiency, attenuation and scatter. Absolute quantification has been demonstrated on the Siemens micro-PET R4, using a reconstruction algorithm implementing corrections for randoms, dead time, geometric effects, attenuation and scatter[6]. In that method a transmission scan was used to derive the attenuation map. A calibration factor for absolute quantification was derived from a scan of a uniform phantom. In this work we present a method for performing absolute quantification on the $\mathrm{LabPET}^{\mathrm{TM}}$ system using CTbased attenuation correction and a reference point source for calibration.

\section{MATERIALS AND METHODS}

\section{A. Equipment}

All acquisitions were done on a GE Triumph (GE Healthcare) system. This tri-modality system consists of a microPET $\left(\mathrm{LabPET}^{\mathrm{TM}}\right)$, micro-CT $\left(\mathrm{X}-\mathrm{O}^{\mathrm{TM}}\right)$ and micro-SPECT $(\mathrm{X}$ SPECT ${ }^{\circledR}$ ) scanner. The X-SPECT ${ }^{\circledR}$ scanner was not used in this work. The LabPET ${ }^{\mathrm{TM}}$ scanner is an APD-based scanner with alternating rings of LYSO and LGSO crystals[7]. The crystals are trapezoidal in shape, with dimensions of $2 \mathrm{x}$ $2 \times 14 \mathrm{~mm}$ (LGSO) and $2 \times 2 \times 12.6 \mathrm{~mm}$ (LYSO). Each APD is coupled to a LYSO and a LGSO crystal. Due to the 
different timing properties of LYSO and LGSO, the origin of a light pulse can be determined from the pulse shape[8]. This leads to effective one-to-one coupling. The crystals and APDs are organized in modules of 4 (tangential) by 2 (axial) crystals. There are 48 modules in the tangential direction and 16 modules in the axial direction. There are gaps between the modules, approximately $2.0 \mathrm{~mm}$ and $0.77 \mathrm{~mm}$ in the tangential and axial direction respectively, as depicted in fig. 1 . In the tangential direction there is also a $0.2 \mathrm{~mm}$ gap between the individual crystals as well. The $\mathrm{X}-\mathrm{O}^{\mathrm{TM}}$ is a micro-CT scanner with adjustable magnification, achieved by moving the position of the object between the X-ray source and detector.

\section{B. Acquisitions}

Two PET experiments were done, summarized in table I. For all PET scans a total acquisition time of 1 hour was used and a single bed position was imaged. The objective of the first experiment was to assess the performance of our method at high and low count rates. Two scans of the same phantom were done. It consisted of a PMMA cylinder filled with water in which 5 vials with different ${ }^{18} \mathrm{~F}$-fluorodeoxyglucose (FDG) activity concentrations were placed. The radius of the vials was $5 \mathrm{~mm}$, the radius of the cylinder was $25 \mathrm{~mm}$. The activity in each of the vials was measured in a dose calibrator (VDC404, Veenstra, Joure, The Netherlands) and ranged from 4 to $25 \mathrm{MBq}$. A first scan at high count rate was done with a total activity inside the scanner of approximately $80 \mathrm{MBq}$. To evaluate the performance at low count rate, a second scan was done 4 hours later, when the total activity had decayed to approximately $20 \mathrm{MBq}$.

As APDs are very sensitive to temperature and other operating condition changes[9], the detection efficiency of the system can change considerably over short time periods (less than a day). It is therefore difficult to calibrate the system once per day or per week and use this calibration for the subsequent scans. Therefore point sources with a known activity were placed inside the field of view (under the bed) during each of the scans. The counts obtained in these point sources can be used to calculate a correction factor for the varying detection efficiency of the scanner. In the first experiment $4{ }^{18} \mathrm{~F}$ point sources were used. The activity inside the point sources was measured in a dose calibrator and ranged from 200 to 300 $\mathrm{kBq}$.

Due to the relatively short half-life of ${ }^{18} \mathrm{~F}$, using such reference point sources requires de novo fabrication of the point sources for each experiment. Using a reference point source with an isotope with a longer half-life would be more practical. The goal of the second experiment was to assess the feasibility of using a reference point source containing ${ }^{22} \mathrm{Na}$. Therefore, one ${ }^{18} \mathrm{~F}$ and one ${ }^{22} \mathrm{Na}$ point source were placed inside the field of view. The activity of the ${ }^{22} \mathrm{Na}$ point source was calibrated by the manufacturer (Eckert \& Ziegler Isotope Products, Berlin, Germany) and was $613 \mathrm{kBq}$ at the time of acquisition. A similar phantom as in the first experiment was scanned, consisting of the same PMMA cylinder filled with water and 3 vials and 1 sphere (radius $2 \mathrm{~mm}$ ). The sphere and vials were filled with ${ }^{18} \mathrm{~F}$-FDG, each containing a total activity between 2 and $9 \mathrm{MBq}$. The total activity in the scanner was approximately $20 \mathrm{MBq}$, as in the second scan of the first experiment.

\begin{tabular}{lllll}
\hline & & Phantom & Total activity & Reference source \\
\hline \hline 1 & A & 5 vials & $80 \mathrm{MBq}$ & ${ }^{18} \mathrm{~F}$ \\
& B & 5 vials & $20 \mathrm{MBq}$ & ${ }^{18} \mathrm{~F}$ \\
2 & & 3 vials +1 sphere & $20 \mathrm{MBq}$ & ${ }^{18} \mathrm{~F}+{ }^{22} \mathrm{Na}$ \\
\hline
\end{tabular}

Table I

SUMMARY OF BOTH MICRO-PET EXPERIMENTS. THE FIRST EXPERIMENT WAS PERFORMED TO EVALUATE THE PERFORMANCE AT HIGH AND LOW COUNT RATES. THE GOAL OF THE SECOND EXPERIMENT WAS TO EVALUATE THE FEASIBILITY OF USING DIFFERENT ISOTOPES IN THE REFERENCE POINT SOURCES.

Before each micro-PET scan a micro-CT was performed by acquiring 1024 projections into a 2368 x 2240 matrix with $50 \mu \mathrm{m}$ pixel size. A magnification of 1.3 was used. The CT settings were $75 \mathrm{kVp}$ and $285 \mu \mathrm{A}$. The micro-CT acquisition time was approximately 9 minutes.

\section{Image reconstruction}

The current reconstruction software available from the scanner manufacturer is a 2D ML-EM algorithm with single slice rebinning (SSRB). It is well known that SSRB generates artefacts for points that are not in the transaxial center. The software provided by the manufacturer also does not include attenuation correction. Therefore we developed our own 3D ML-EM reconstruction algorithm including corrections for the gaps between modules, geometric sensitivity, random coincidences and photon attenuation. Scatter correction was not implemented as it was deemed to have a small effect in micro-PET of relatively small objects[10]. The correction for detection efficiency is performed after the reconstruction and is described in the next section.

The micro-PET data acquired in all scans were reconstructed to a $80 \times 80 \times 64$ matrix with $1 \mathrm{~mm}$ isotropic voxel size. The reconstruction is based on the Line Of Response (LOR) histogram. A LOR histogram contains the number of measured counts for each crystal pair. An estimation of random coincidences is obtained using a delayed coincidence window. The LOR histogram that is used for reconstruction is obtained by subtracting the random histogram from the prompt histogram. This yields the estimated true histogram.

To determine the LOR along which the forward and back projection needs to be performed, the three-dimensional position of each of the crystals is calculated for each histogram bin. To avoid sampling artefacts, random sampling on the crystal surface is used to calculate the position of the end points of the LOR. Four rays were sampled randomly for each histogram bin. This random sampling is repeated in each iteration. Sampling over the actual crystal surface would lead to artefacts in the reconstructed images, due to the gaps between the crystals and modules which amount to almost 25 $\%$ of the total surface of the scanner. We have corrected for this effect by performing random sampling on a region larger than the crystal surface, as shown in fig. 2. As the crystals 


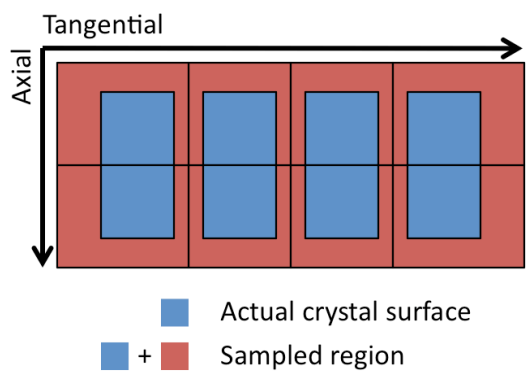

Figure 2. Illustration of the gap correction for a single module. The actual crystal surface is depicted in blue. The position on the crystal surface is randomly sampled from the red + blue region. This yields a scanner with virtually no gaps.

at the tangential border of a module are made virtually larger than the others with this method, the number of counts in these crystals was multiplied with a correction factor. This correction factor is calculated as the ratio of the sampled surface in these crystals to the sampled surface in the other crystals. This method is a form of nearest neighbor interpolation of the data that was lost inside the gaps. The calculated points then determine the line along which the number of counts in the LOR histogram bin are forward and back projected.

Although the gaps are corrected for, correction for geometric sensitivity is still necessary, as the solid angle coverage of the scanner will still vary between points inside the field of view. A geometric sensitivity map was calculated by performing a single back projection of all available LORs[11]. Crystals which are defective and are not measuring at the time of the scan do not contribute to the geometric sensitivity of the scanner. LORs containing these crystals should therefore also not be included in the geometric sensitivity, and should not be back projected in the calculation of the sensitivity map. The active crystals were determined from a normalization scan with a ${ }^{68} \mathrm{Ge}$ rod source performing a full rotation close to the crystal surfaces. All crystals should be irradiated by the normalization source and hence all crystals should register some counts during the normalization scan. Crystals which have not acquired any counts during the normalization scan are considered to be offline and are excluded from the geometric sensitivity calculation.

\section{Attenuation correction}

Non-uniform attenuation correction was performed using an attenuation map derived from the micro-CT image, as described before by Chow et al[12]. The micro-CT images were first reconstructed with the reconstruction software provided by the manufacturer. The reconstructed matrix was $512 \times 512 \mathrm{x}$ 512 with $0.25 \mathrm{~mm}$ voxel size. The images were downsampled to a $80 \times 80 \times 64$ matrix with $1 \mathrm{~mm}$ voxel size by summing together neighboring voxels. Then a conversion from microCT values to $511 \mathrm{keV}$ attenuation coefficients was done using bilinear scaling[13]:
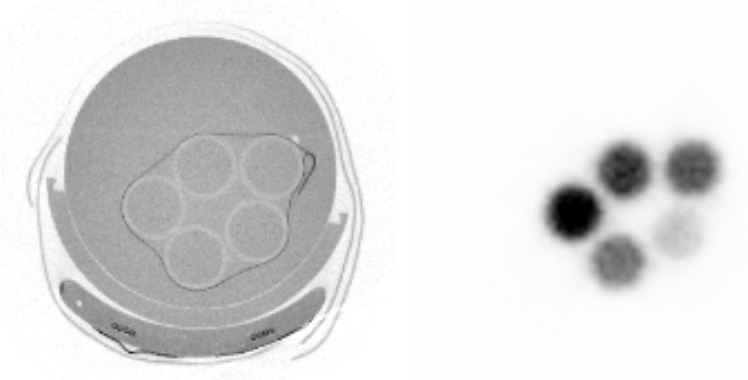

Figure 3. Transverse slice of the micro-CT (left) and micro-PET (right) image of the second acquisition of the first experiment.

$$
\begin{aligned}
\mu\left(\mathrm{cm}^{-1}\right) & =0.097 \frac{I}{I_{\text {water }}} & & I \leq I_{\text {water }} \\
& =0.097+0.096 \frac{I-I_{\text {water }}}{I_{\text {water }}} & & I>I_{\text {water }}
\end{aligned}
$$

In this equation $I$ is the $\mathrm{CT}$ image intensity in the voxel and $I_{\text {water }}$ is the image intensity in a voxel containing water, which was obtained from a micro-CT scan of a uniform water phantom.

\section{E. Calculation of absolute activity}

In the reconstructed images volumes of interest (VOIs) were drawn over all point sources. For each point source a correction factor for the absolute sensitivity was calculated. This was done by calculating the ratio of the actual activity in the point source to the reconstructed activity in the point source:

$$
F_{a b s}=\frac{A_{P S}}{N_{P S}}
$$

where $A_{P S}$ is the activity measured in the dose calibrator (in $\mathrm{Bq}$ ) and $N_{P S}$ is the total number of counts in the VOI over the point source in the reconstructed image. The absolute activity in a VOI can then be calculated as follows:

$$
C_{V O I}=N_{V O I} F_{a b s} \frac{D_{V O I}}{D_{P S}}
$$

with $C_{V O I}$ the calculated activity in the VOI, $N_{V O I}$ the number of counts in the VOI in the reconstructed image and $F_{a b s}$ the correction factor for absolute sensitivity. The last factor is a correction factor for decay. This factor is only needed if a different isotope is used in the reference point source and the VOI. Both $D_{V O I}$ and $D_{P S}$ are calculated using the following equation:

$$
D_{X}=\left(\int_{T_{s}}^{T_{e}} e^{\frac{-\ln (2) t}{t_{1 / 2, X}}} d t\right)^{-1}
$$

Where $t_{1 / 2, X}$ is the half life of the isotope in either the point source or the VOI, $T_{s}$ and $T_{e}$ are the start and end times of the scan and the time of measurement in the dose calibrator is assumed to be 0 . This means that equation 3 calculates the absolute activity level at the time of measurement in the dose calibrator. 


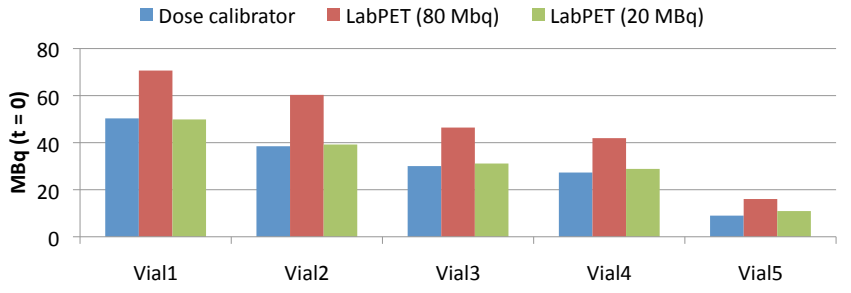

Figure 4. Calculated total activity in the images obtained from both scans of the first experiment (high and low count rate) compared to the activity measured in the dose calibrator. The activity was calculated using the average correction factor for absolute sensitivity of the $4{ }^{18} \mathrm{~F}$ point sources.

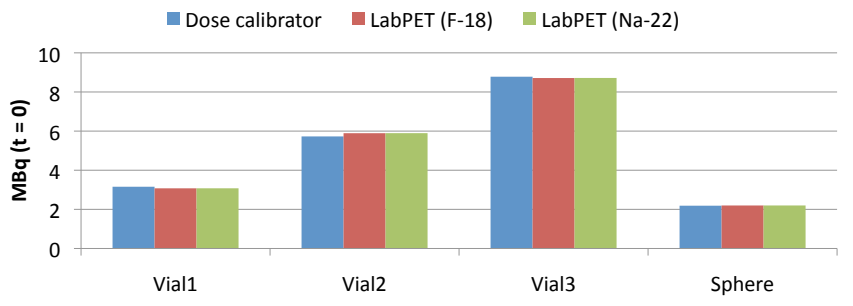

Figure 5. Calculated total activity in the images obtained from the second experiment compared to the activity measured in the dose calibrator. The activity was calculated using two correction factors for absolute sensitivity: based on the ${ }^{18} \mathrm{~F}$ point source and based on the ${ }^{22} \mathrm{Na}$ point source.

\section{F. Evaluation}

VOIs were drawn over the vials in the images of the first experiment and over the vials and the sphere in the images of the second experiment. The total activity in the vials in the two acquisitions of the first experiment was calculated using eq. 3, where the average of the $F_{a b s}$ calculated for all 4 point sources was used as correction factor. For the second experiment the total activity in the vials and the sphere was calculated twice: once using the correction factor of the ${ }^{18} \mathrm{~F}$ point source and once using the correction factor of the ${ }^{22} \mathrm{Na}$ point source. The calculated total activities were compared to the measured total activity in the dose calibrator by calculating the relative difference:

$$
\Delta_{i}(\%)=\frac{C_{i}-A_{i}}{A_{i}}
$$

where $C_{i}$ is the calculated activity in the vial obtained from the reconstructed micro-PET image using eq. 3 and $A_{i}$ is the actual activity in the vial, measured with the dose calibrator.

\section{RESULTS}

Fig. 3 depicts a transverse slice of the micro-CT and the reconstructed PET image of the second scan. Fig. 4 shows the calculated activity in the vials in the first experiment compared to the activity measured in the dose calibrator. Fig. 5 shows the same for the vials and the sphere and for both correction factors used for the second experiment. The average quantification error was $56 \%$ and $6.4 \%$ for the first and second scan respectively and $0.2 \%$ and $0.6 \%$ for the third scan with ${ }^{18} \mathrm{~F}$ and ${ }^{22} \mathrm{Na}$ correction factor respectively.

\section{Discussion}

The quantification error in the first scan of the first experiment is too large. This is probably caused by count rate effects. As APDs are inherently slow, APD-based systems such as the $\mathrm{LabPET}^{\mathrm{TM}}$ system require a wide coincidence window as well as a wide energy window. As the timing properties of LYSO and LGSO are different, different coincidence windows are set for LGSO-LGSO, LYSO-LYSO and LGSOLYSO coincidences, ranging from 10 to $20 \mathrm{~ns}$. These large coincidence windows lead to high random coincidence rates at high count rates, which are difficult to correct accurately. $80 \mathrm{MBq}(\approx 2 \mathrm{mCi})$ can be considered a high count rate for a small animal PET system. Therefore, the large errors seen in the first scan can be caused by random coincidences. In the second scan of the first experiment and in the second experiment, where only $20 \mathrm{MBq}(\approx 0.5 \mathrm{mCi})$ was in the scanner, the quantification error is much smaller. Therefore one should always aim for a total activity of maximum 20 $\mathrm{MBq}$ at the start of the acquisition.

There is no difference between the accuracy of quantification based on ${ }^{18} \mathrm{~F}$ or ${ }^{22} \mathrm{Na}$ point sources. This means that the $1274.5 \mathrm{keV}$ gamma photon which is emitted together with each ${ }^{22} \mathrm{Na}$ decay does not cause any problems. This is probably due to the fact that the energy of the gamma photon is well above the energy threshold of the scanner. As ${ }^{18} \mathrm{~F}$ has a half life of 110 minutes, reference point sources containing ${ }^{18} \mathrm{~F}$ need to be fabricated at least daily and probably even twice per day. This imposes an extra effort on experiments. As ${ }^{22} \mathrm{Na}$ has a much longer decay time (2.6 years), the use of ${ }^{22} \mathrm{Na}$ is much more practical as the same reference point source can be used for several years.

Another disadvantage of using self-made ${ }^{18} \mathrm{~F}$ point sources is that their activity needs to be measured in a dose calibrator, which is often not very accurate for low activities $(\leq 5 \mu \mathrm{Ci})$. A possible solution for this is to prepare the ${ }^{18} \mathrm{~F}$ point sources with a higher activity level a few hours before the experiment. However, accurately calibrated ${ }^{22} \mathrm{Na}$ point sources can also be purchased. This offers a more practical solution.

\section{CONCLUSION}

The feasibility of absolute quantification on the LabPET $^{\mathrm{TM}}$ system was demonstrated. Images were reconstructed using a $3 \mathrm{D}$ ML-EM reconstruction implementing gap correction, geometric sensitivity correction and micro-CT based attenuation correction. A correction factor for the absolute sensitivity of the system was determinated using ${ }^{18} \mathrm{~F}$ or ${ }^{22} \mathrm{Na}$ point sources. When the count rate is not too high, corresponding to a total activity of approximately $20 \mathrm{MBq}$, absolute quantification is possible with an average quantification error smaller than $6.4 \%$. At higher count rates, the quantification error increases quickly, leading to an average quantification error larger than $50 \%$ when $80 \mathrm{MBq}$ is present in the scanner.

\section{ACKNOWLEDGEMENTS}

This work was supported by the EU FP7 projects HYPERimage (Grant Agreement Nr 201651) and SUBLIMA 
(Grant Agreement Nr 241711). Roel Van Holen is supported by a postdoctoral fellowship of the Research Foundation Flanders (FWO). Christian Vanhove is supported by the GROUP-ID consortium from Ghent University.

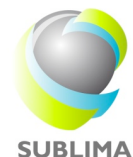

\section{REFERENCES}

[1] H Young, R Baum, U Cremerius, K Herholz, O Hoekstra, A A Lammertsma, J Pruim, and P Price, "Measurement of clinical and subclinical tumour response using [18f]-fluorodeoxyglucose and positron emission tomography: review and 1999 eortc recommendations. european organization for research and treatment of cancer (eortc) pet study group," Eur J Cancer, vol. 35, no. 13, pp. 1773-82, Dec 1999.

[2] L Chaiken, S Rege, C Hoh, Y Choi, B Jabour, G Juillard, R Hawkins, and R Parker, "Positron emission tomography with fluorodeoxyglucose to evaluate tumor response and control after radiation therapy," Int $J$ Radiat Oncol Biol Phys, vol. 27, no. 2, pp. 455-64, Sep 1993.

[3] PD Acton, H Zhuang, and A Alavi, "Quantification in pet," Radiol Clin North Am, vol. 42, no. 6, pp. 1055-62, viii, Nov 2004.

[4] Jason S Lewis, Judith M Connett, Joel R Garbow, Thomas L Buettner, Yasuhisa Fujibayashi, James W Fleshman, and Michael J Welch, "Copper-64-pyruvaldehyde-bis(n(4)-methylthiosemicarbazone) for the prevention of tumor growth at wound sites following laparoscopic surgery: monitoring therapy response with micropet and magnetic resonance imaging," Cancer Res, vol. 62, no. 2, pp. 445-9, Jan 2002.

[5] A H Moore, C L Osteen, A F Chatziioannou, D A Hovda, and S R Cherry, "Quantitative assessment of longitudinal metabolic changes in vivo after traumatic brain injury in the adult rat using fdg-micropet," $J$ Cereb Blood Flow Metab, vol. 20, no. 10, pp. 1492-501, Oct 2000.

[6] Paul Vaska, Daniel J Rubins, David L Alexoff, and Wynne K Schiffer, "Quantitative imaging with the micro-pet small-animal pet tomograph," Int Rev Neurobiol, vol. 73, pp. 191-218, Jan 2006.

[7] M Tetrault, N Viscogliosi, J Riendeau, F Bélanger, J Michaud, H Semmaoui, P Bérard, F Lemieux, L Arpin, M Bergeron, J Cadorette, CM Pepin, G Robert, M Lepage, R Lecomte, and R Fontaine, "System architecture of the labpet ${ }^{\mathrm{TM}}$ small animal pet scanner," IEEE T Nucl Sci, Jan 2008.

[8] N Viscogliosi, J Riendeau, P Berard, and M Tetrault, "Real time implementation of a wiener filter based crystal identification algorithm," IEEE T Nucl Sci, vol. 55, no. 3, pp. 925 - 929, 2008.

[9] B Pichler, A Kolb, T Nägele, and H Schlemmer, "Pet/mri: paving the way for the next generation of clinical multimodality imaging applications," J Nucl Med, vol. 51, no. 3, pp. 333-6, Mar 2010.

[10] A Konik, T Koesters, M Madsen, and J Sunderland, "Evaluation of attenuation and scatter correction requirements as a function of object size in small animal pet imaging," IEEE T Nucl Sci, vol. Accepted for publication, 2011.

[11] J Qi, "Calculation of the sensitivity image in list-mode reconstruction for pet," IEEE T Nucl Sci, vol. 53, no. 5, pp. 2746 - 2751, 2006.

[12] P Chow, F Rannou, and A Chatziioannou, "Attenuation correction for small animal pet tomographs," Phys Med Biol, vol. 50, pp. 1837-50, Jan 2005.

[13] C Burger, G Goerres, S Schoenes, and A Buck, "Pet attenuation coefficients from ct images: experimental evaluation of the transformation of ct into pet 511-kev attenuation coefficients," Eur J Nucl Med, Jan 2002. 